\title{
Solar irradiances measured using SPN1 radiometers: uncertainties and clues for development
}

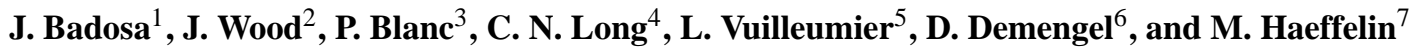 \\ ${ }^{1}$ Laboratoire de Météorologie Dynamique, Palaiseau, France \\ ${ }^{2}$ Peak Design, Winster, UK \\ ${ }^{3}$ MINES ParisTech, PSL Research University, O.I.E. - Centre Observation, Impacts, Energie, \\ CS 10207 rue Claude Daunesse 06904 Sophia Antipolis Cedex, France \\ ${ }^{4}$ Pacific Northwest National Laboratory, Richland, WA, USA \\ ${ }^{5}$ Meteoswiss, Payerne, Switzerland \\ ${ }^{6}$ EDF R\&D-CEREA, Chatou, France \\ ${ }^{7}$ Institut Pierre Simon Laplace (IPSL), Palaiseau, France \\ Correspondence to: J. Badosa (jordi.badosa@lmd.polytechnique.fr)
}

Received: 3 June 2014 - Published in Atmos. Meas. Tech. Discuss.: 6 August 2014

Revised: 19 October 2014 - Accepted: 5 November 2014 - Published: 8 December 2014

\begin{abstract}
The fast development of solar radiation and energy applications, such as photovoltaic and solar thermodynamic systems, has increased the need for solar radiation measurement and monitoring, for not only the global but also the diffuse and direct components. End users look for the best compromise between getting close to state-of-the-art measurements and keeping low capital, maintenance and operating costs. Among the existing commercial options, SPN1 is a relatively low cost solar radiometer that estimates global and diffuse solar irradiances from seven thermopile sensors under a shading mask and without moving parts.

This work presents a comprehensive study of SPN1 accuracy and sources of uncertainty, drawing on laboratory experiments, numerical modelling and comparison studies between measurements from this sensor and state-of-the art instruments for six diverse sites. Several clues are provided for improving the SPN1 accuracy and agreement with state-ofthe art measurements.
\end{abstract}

\section{Introduction}

Developments in the use of renewable solar energy have increased the need for solar radiation measurement of global horizontal irradiance (GHI), direct normal irradiance (DNI) and diffuse horizontal irradiance (DHI) to characterize the solar resource. These in situ pyranometric measurements are essential for region- or site-specific solar resource assessment, for the monitoring of solar power plants, and also for some short-term forecasting, for example for electricity grid integration. Whether for solar resource assessment at a specific site, at short timescales, or for a long-term analysis of spatial and temporal variability with a network of pyranometric sensors, all these applications require reliable continuous measurements.

The current state-of-the-art measurement uses a pyrheliometer on a solar tracker to measure DNI, and pyranometers (one shaded by a tracker-mounted ball) to measure respectively the GHI and DHI. Such instruments are used for example by the Baseline Surface Radiation Network (BSRN; MacArthur, 2005), but incur high capital and maintenance costs and require frequent and complex human maintenance on site.

Other instruments for measuring the global, diffuse and direct components are as follows:

1. pyranometer for GHI, and pyranometer with shade ring for DHI. DNI can be calculated from these two components. The shade ring must be regularly manually adjusted for changes in solar declination, and a correction must be applied for the shaded part of the diffuse sky (WMO, 2010). 
2. rotating shadow-band irradiometers (RSIs). These use a silicon photodiode detector, and a motorized rotating shading ring to measure both the GHI and DHI. Further corrections must be applied to correct for the photodiode spectral response, cosine response and thermal response to give more accurate measurements (e.g. Geuder et al., 2003).

All of these techniques require moving parts that must be kept accurately aligned, either automatically or manually, which increases the continuing maintenance cost.

The SPN1 (Wood, 1999) is a radiometer without moving parts that measures GHI and DHI total short-wave irradiance (from 400 to $2700 \mathrm{~nm}$ ) expressed in $\mathrm{W} \mathrm{m}^{-2}$. It also gives a sunshine status. The manufacturer specifies an overall accuracy for both the GHI and DHI of $\pm 8 \%\left( \pm 10 \mathrm{~W} \mathrm{~m}^{-2}\right)$ for individual readings, which is close to the "Good Quality Pyranometer" classification by the World Meteorological Organization (WMO). Table 1 summarizes nominal SPN1 specifications as given by Delta-T Devices Ltd (2007).

SPN1 was designed with seven thermopiles: six sensors placed on a hexagonal grid, one sensor at the centre, under a complex static shading mask, in such a way to ensure that, at any time, for any location:

- at least one sensor is always exposed to the full solar beam

- at least one sensor is always completely shaded

- the solid angle of the shading mask is equal to pi, thus corresponding to half the hemispherical solid angle.

Under the assumption of isotropic diffuse sky radiance, the third property related to the shading mask implies that all sensors receive equal amounts $(50 \%)$ of diffuse irradiance from the rest of the sky hemisphere.

Figure 1 shows the parts of the sky hemisphere seen by the different sensors plotted using an equiangular projection, where radial distance on the image represents zenith angle on the hemisphere (Long et al., 2010).

DHI and GHI measurements are retrieved using a simple principle. At every measuring moment, let MIN and MAX be the minimum and maximum signals measured among the seven sensors.

For the diffuse, SPN1 processing assumes that the half of the sky that is not seen by each sensor is exactly the same as the half that it does see.

From this,

$$
\begin{aligned}
& \mathrm{DHI}=2 \times \mathrm{MIN} \\
& \mathrm{BHI}=(\mathrm{MAX}-\mathrm{MIN}) \\
& \mathrm{GHI}=\mathrm{BHI}+\mathrm{DHI}=\mathrm{MAX}+\mathrm{MIN} .
\end{aligned}
$$

After this, some other calibration based on linear adjustments is applied (see the details in the Supplement).

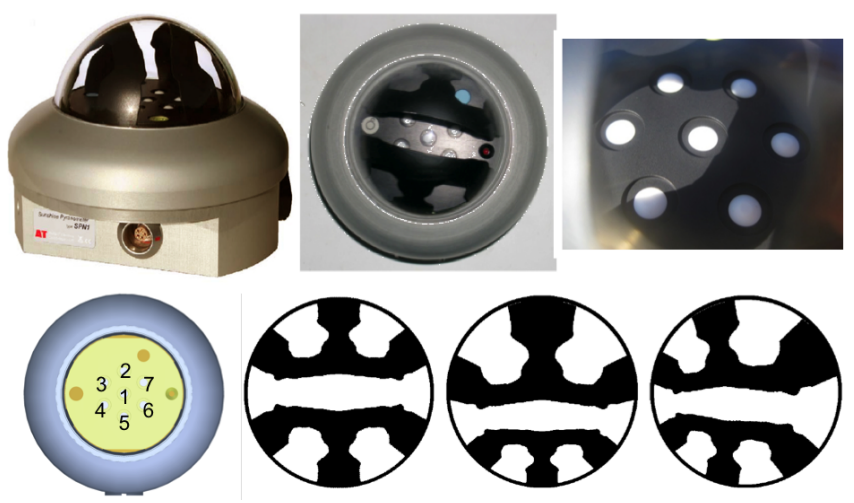

Figure 1. Top row: different SPN1 views; photo on the right shows a shadow pattern on the seven sensors for a particular sunny moment. Bottom row: SPN1 detector numbering; sky seen under shade patterns as seen for sensor 1 (left), sensors 2 and 5 (middle) and sensors 3, 4, 6, 7 (right). Sources: Long et al. (2010) and Delta-T Devices Ltd. (2007).

It is necessary to notice that the sensors measuring MIN and MAX will be different at different times, and this is not trackable when using the current commercial firmware as the knowledge of the SPN1's azimuthal position is not required. However, it is to be noted that this azimuthal information can be retrieved from the analysis of the individual measurements from the seven thermopiles available through an RS232 port.

DNI is calculated afterwards from the GHI and DHI values, as follows:

$\mathrm{DNI}=(\mathrm{GHI}-\mathrm{DHI}) / \cos (\mathrm{SZA})$,

where SZA is the solar zenith angle.

More detailed technical information about SPN1 is found in its User Manual (Delta-T Devices Ltd, 2007).

SPN1 first came out about 10 years ago, and since then, its use for on-site solar resource assessment and its presence in radiation measurement networks have been growing, notably for industries in the domain of solar energy. As a consequence, the number of studies using, evaluating and comparing this sensor with other available types is increasing, although the number of related scientific publications is still low. Psiloglou et al. (2013) compared GHI and DHI measurements from SPN1 sensors against classical unshaded and ring-shaded pyranometer measurements at Penteli, Greece, during Summer 2011. They stated that accuracies were easily verified for $1 \mathrm{~min}$ measurements of GHI, with root mean square error (RMSE) values varying between 2.6 and $3.6 \%$, whereas for the DHI radiation, the observed RMSEs (10.8$17.0 \%$ ) were not compatible with stated accuracies. They proposed new uncertainty values for the diffuse component and suggested that a correction factor should be used to improve its accuracy.

Myers and Wilcox (2009) presented the results of a comparison study of several radiometers at the US National Re- 
Table 1. SPN1 nominal specifications (Delta-T Devices Ltd, 2007).

\begin{tabular}{|c|c|}
\hline Overall accuracy: total (global) and diffuse radiation & $\begin{array}{l} \pm 5 \% \text { daily integrals } \\
\pm 5 \% \pm 10 \mathrm{~W} \mathrm{~m}^{-2} \text { hourly averages } \\
\pm 8 \% \pm 10 \mathrm{~W} \mathrm{~m}^{-2} \text { individual readings }\end{array}$ \\
\hline Cosine response accuracy & $\pm 2 \%$ of incoming radiation over $0-90^{\circ}$ zenith angle \\
\hline Azimuth angle accuracy & $\pm 5 \%$ over $360^{\circ}$ rotation \\
\hline Temperature coefficient & $\pm 0.02 \%$ per ${ }^{\circ} \mathrm{C}$ typical \\
\hline Spectral sensitivity variation & $10 \%$ typical \\
\hline Non-linearity & $<1 \%$ \\
\hline Zero offsets & $\begin{array}{l}<3 \mathrm{~W} \mathrm{~m}^{-2} \text { for a change of } 5^{\circ} \mathrm{Ch}^{-1} \text { in ambient temperature } \\
<3 \mathrm{~W} \mathrm{~m}^{-2} \text { dark reading }\end{array}$ \\
\hline
\end{tabular}

newable Energy Laboratory (NREL) Solar Radiation Research Laboratory (SRRL) in Golden, Colorado. They computed biases from the comparison, which were described as a function of SZA. A bias between -3.7 and $-0.7 \%$ was found for GHI, but for DHI larger bias between -13.8 and $-4.3 \%$ was observed. Myers (2010) found SPN1 DNI measurement differences (with respect to the reference DNI) of \pm 15 to $20 \%$, especially at DNIs below $400 \mathrm{~W} \mathrm{~m}^{-2}$. At higher DNIs, above $600 \mathrm{~W} \mathrm{~m}^{-2}$, useful for concentrating solar applications, the SPN1 horizontal radiometers produced DNI estimates with uncertainty comparable to rotating shadow-band radiometer (RSR) DNI estimates $( \pm 10 \%)$ for both $1 \mathrm{~min}$ and hourly averaged data.

The present study aims to list and quantify comprehensively the uncertainty sources for the GHI, DHI and DNI measurements when using SPN1. In particular, comparisons are made with tracker-based measurements (TBMs) using pyranometers for the GHI and DHI measurements and a pyrheliometer for DNI, which represent the state of the art for these measurements. For this, SPN1 and TBM data from six sites have been considered, together with laboratory and other ancillary measurements, some of which are original to this paper as radiative measurements.

\section{Materials and methods}

GHI and DHI measurements and computed DNI from SPN1 sensors with concurrent TBMs as a reference were considered for six sites with different geographical and meteorological contexts: four mid-latitude sites (Winster in England; NREL's Solar Radiation Research Laboratory, Golden, CO, USA; SIRTA atmospheric observatory, Palaiseau, France; MeteoSwiss Aerological station, Payerne, Switzerland) and two tropical sites (Addu Atoll in Maldives and Roseraye in Reunion Island). DNI for the SPN1 is computed from the 1 min averages of GHI and DHI, using Eq. (4). Table 2 summarizes the geo-location of the "sites", the data temporal coverage, the instruments used and data volume descriptions.
Data from days for which operation failures can be identified (either from maintenance log-books or visual inspection of the data) were removed from the study. This corresponds to 28 days in Palaiseau (22 of these were affected by time stamp drift error for SPN1 data acquisition), 4 days in Golden, and 15 days in Roseraye (due to a cyclonic episode that made TBM unavailable for safety reasons).

For Payerne, the data used are part of the DNI-comparison campaign organized in the frame of the EU-funded COST Action ES1002 Weather Intelligence for Renewable Energies (WIRE). Three SPN1 radiometers have been installed for this campaign together with two triplets of rotating shadowband radiometers from different manufacturers. Available data were already partly quality controlled, in particular for DNI reference measurements, resulting in $5.6 \%$ of data left out in the considered period for SZA $<80$ (see Table 2).

All remaining data were further tested using the Baseline Surface Radiation Network (BSRN) recommended quality control (QC) tests (Roesch et al., 2011). Three tests for GHI, DHI and DNI measurements were performed: extremely rare limits (ERL) test which sets physical upper and lower limit bounds to each quantity and two consistency tests: DHI/GHI (Diffuse ratio) and the ratio of GHI over calculated global from DHI and DNI (GHI/GHI*, where $\left.\mathrm{GHI}^{*}=\mathrm{DNI} \cdot \cos (\mathrm{SZA})+\mathrm{DHI}\right)$. This latter can only be applied to TBM since independent GHI, DHI, DNI measurements are required. The percentages of data passing the tests for SZA $<80$ are separately given for TBM and SPN1 in Table 3 .

The final considered data sets for each site were built from all $1 \mathrm{~min}$ data for which all tests (ERL and DHI / DHI for TBM and SPN1 and GHI/GHI* for TBM) were passed. Data were further limited to SZA lower than $80^{\circ}$ and both GHI and DHI measurements larger than $5 \mathrm{~W} \mathrm{~m}^{-2}$. Moreover, for Palaiseau, all data for solar azimuth angle (SAA) lower than $70^{\circ}$ (early morning summer data, with $\mathrm{SAA}=180$ for solar noon) were removed due to direct shadow effects on SPN1 from a surrounding meteorological mast. 
Table 2. Sites, data periods and instrumental information.

\begin{tabular}{|c|c|c|c|c|c|}
\hline Site & Latitude/Longitude/Altitude & $\begin{array}{l}\text { Data } \\
\text { period }\end{array}$ & $\begin{array}{l}\text { TBM instruments } \\
\text { (GHI/DHI/DNI) }\end{array}$ & $\begin{array}{l}\text { Sampling/Storing } \\
\text { times }\end{array}$ & $\begin{array}{l}\text { SPN1 } \\
\text { orientation }\end{array}$ \\
\hline Winster & $53.142^{\circ} \mathrm{N} / 1.636^{\circ} \mathrm{W} / 237 \mathrm{~m}$ & Feb 2013-Oct 2013 & K\&Z CMP6/CMP6/CHP1 & $1 \mathrm{~s} / 1 \mathrm{~min}$ & $81^{\circ}$ \\
\hline Palaiseau & $48.712^{\circ} \mathrm{N} / 2.208^{\circ} \mathrm{E} / 165 \mathrm{~m}$ & Dec 2010-Apr 2013 & K\&Z CMP22/CMP22/CH1 & $1 \mathrm{~s} / 1 \mathrm{~min}$ & \\
\hline Payerne & $46.815^{\circ} \mathrm{N} / 6.944^{\circ} \mathrm{E} / 491 \mathrm{~m}$ & Jun 2012-Sep 2013 & K\&Z CMP22/CMP22/CHP1 & $1 \mathrm{~s} / 1 \mathrm{~min}$ & \\
\hline Golden & $39.742^{\circ} \mathrm{N} / 105.18^{\circ} \mathrm{W} / 1829 \mathrm{~m}$ & Jan 2012-Oct 2013 & K\&ZCM22/CM22/CH1 & $1 \mathrm{~s} / 1 \mathrm{~min}$ & \\
\hline Addu Atoll & $0.688^{\circ} \mathrm{S} / 73.501^{\circ} \mathrm{E} / 2 \mathrm{~m}$ & Sep 2011-Feb 2012 & Eppley PSP/8-48/NIP & $1 \mathrm{~s} / 1 \mathrm{~min}$ & \\
\hline Roseraye & $21.137^{\circ} \mathrm{S} / 55.791^{\circ} \mathrm{E} / 135 \mathrm{~m}$ & Jul 2012-May 2013 & K\&Z CMP21/CMP21/CHP1 & $10 \mathrm{~s} / 1 \mathrm{~min}$ & $90^{\circ}$ \\
\hline
\end{tabular}

CF estimations were available for four sites. For Palaiseau and Golden, these were taken from the images of a co-located TSI (Yankee Environmental Systems); for Winster and Addu Atoll, the fractional sky cover (referred to as CF in this paper) was estimated by using the TBMs as inputs to the Long et al. (2006) algorithm.

\section{Quantitative criteria for comparison}

The following metrics definitions (where STDE is standard deviation of the errors, rRMSE is relative RMSE, MAE is mean absolute error, rMAE is relative MAE and MBE is mean bias error) will be used in the present work to describe the absolute and relative dispersion and bias errors:

$$
\begin{aligned}
\mathrm{Avg} & =\frac{\sum_{t=1}^{N} I_{\mathrm{TBM}}}{N} \\
\mathrm{STDE} & =\sqrt{\frac{\sum_{t=1}^{N}\left(I_{\mathrm{SPN} 1}-I_{\mathrm{TBM}} \cdot \text { slope }^{2}\right.}{N}} \\
\mathrm{RMSE} & =\sqrt{\frac{\sum_{t=1}^{N}\left(I_{\mathrm{SPN} 1}-I_{\mathrm{TBM}}\right)^{2}}{N}} \\
\mathrm{rRMSE} & =\frac{\operatorname{RMSE}}{\operatorname{Avg} 100} \\
\mathrm{MAE} & =\frac{\sum_{t=1}^{N}\left|I_{\mathrm{SPN} 1}-I_{\mathrm{TBM}}\right|}{N} \\
\mathrm{rMAE} & =\frac{\operatorname{MAE}_{\mathrm{Avg}} 100}{\mathrm{MBE}}=\frac{\sum_{t=1}^{N}\left(I_{\mathrm{SPN} 1}-I_{\mathrm{TBM}}\right)}{N} \\
\mathrm{rMBE} & =\frac{\operatorname{MBE} 100 .}{\operatorname{Avg}} 100
\end{aligned}
$$

I represents GHI, DHI or DNI. $N$ is the total number of data points considered (values in Table 3); "slope" represents the slope values of the linear regression shown in Fig. 2 and Table 4.

\section{General SPN1 vs. tracker-based measurements agreements}

\subsection{Slope comparison}

General SPN1 vs. TBM comparison can be seen through scatter plots in Fig. 2, where colour scale accounts for the density of points in logarithmic scale. High correlations are seen in all cases with regression coefficients greater than 0.99. Slopes are computed with least-square linear fits (forced through 0, see Fig. 2 and Table 4) and range from 0.955 to 1.021 for $\mathrm{GHI}$, from 0.901 to 0.976 for DHI and from 1.009 to 1.076 for DNI. STDE around the fitting line (as a percentage with respect to the mean TBM value) are also shown in Fig. 2 and range from 3.4 to $4.5 \%$ for GHI, 7.4 to $9.3 \%$ for DHI and 8.7 to $14 \%$ for DNI.

Figure 2 and Table 4 show that DHI is systematically underestimated for all sites and, consequently, DNI is overestimated. DHI data points in Fig. 2 are more dispersed and the STDE values are double with respect to GHI; consequently, so are the STDE values for DNI. These effects are discussed in detail in Sect. 5.

Table 4 also shows the slope values found for DHI and DNI after correcting SPN1 data for the calibration slope found for the GHI (to represent an accurate on-site recalibration). These values are $<1$ for DHI and $>1$ for DNI at all stations (still showing DHI underestimations from SPN1). Winster, Palaiseau, Payerne and Golden show similar DHI slope values (0.937-0.956) while these are considerably lower for Rosearye (0.922) and Addu Atoll (0.903). These two oceanic sites also show significantly higher average DHI values (Table 6) than the other sites. Marine aerosols typically cause more scatter, and hence a larger solar aureole, than landbased aerosols (Gueymard, 2001), and this will increase the average DHI, as will a greater proportion of bright cloudy 

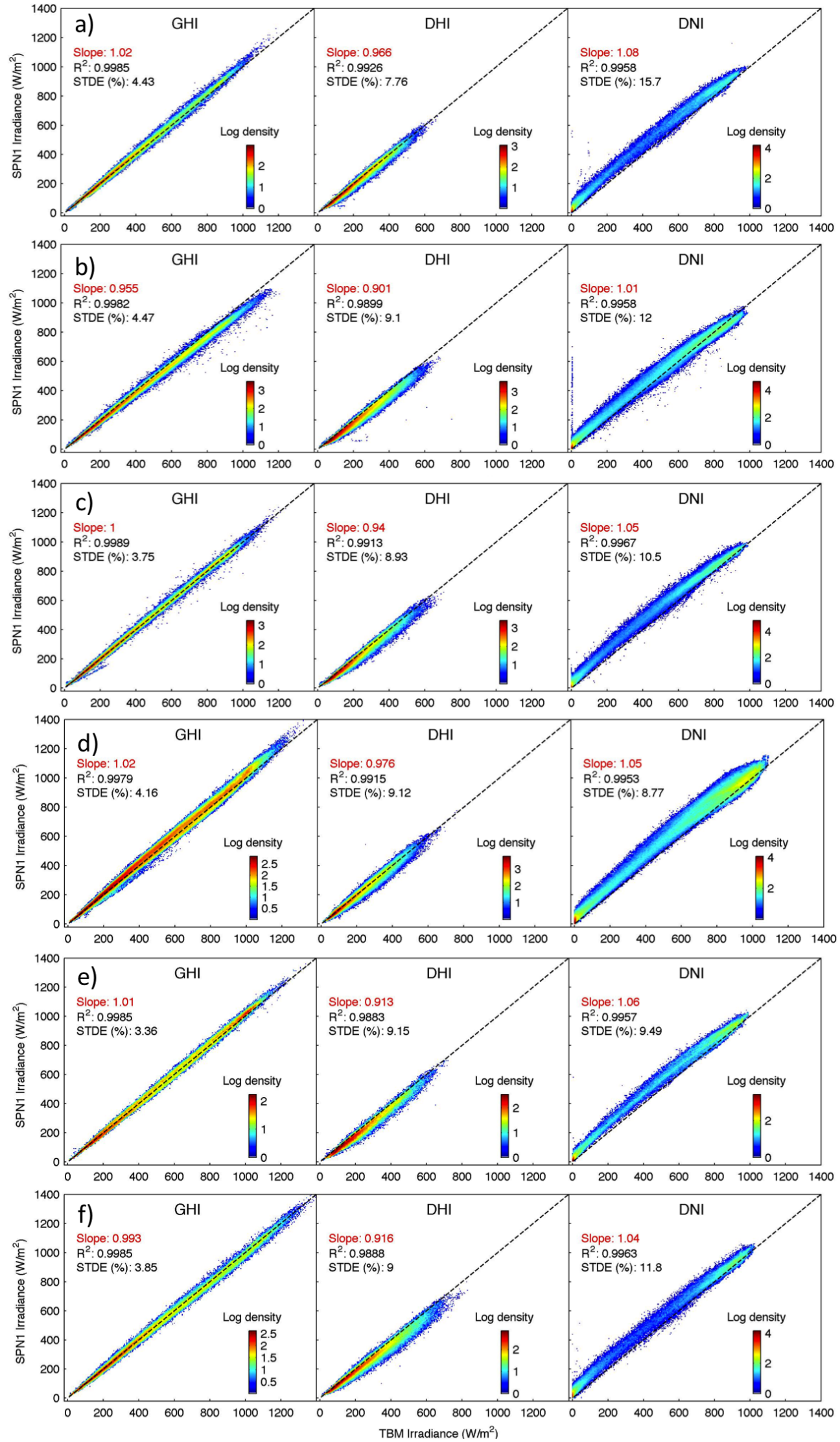

Figure 2. SPN1 vs. TBM measurements for GHI, DHI and DNI at (a) Winster, (b) Palaiseau, (c) Payerne, (d) Golden, (e) Addu Atoll and (f) Roseraye. 
Table 3. Data percentage (with respect to the initial data volume for $\mathrm{SZA}<80^{\circ}$ ) of data not passing BSRN quality control recommended tests v2 for GHI, DHI and DNI measurements - ERL, diffuse ratio test (DHI / GHI) and the ratio of GHI to calculated global from DHI and DNI (GHI / GHI*). The percentages of data passing the tests for SZA $<80$ are separately given for TBM and SPN1 and the total volume of data of SPN1 and TBM concurrent good data (and having GHI and DHI $<5 \mathrm{~W} \mathrm{~m}^{-2}$ ) for each site is found in the last column.

\begin{tabular}{|c|c|c|c|c|c|c|c|c|}
\hline & \multicolumn{4}{|c|}{ TBM } & \multicolumn{3}{|c|}{ SPN1 } & \multirow{2}{*}{$\begin{array}{r}N \text { data } \\
\text { Final data volume } \\
(\% \text { of initial volume })\end{array}$} \\
\hline & $\begin{array}{l}\% \text { ERL } \\
(\text { (GHI/DHI/DNI) }\end{array}$ & $\% \mathrm{DHI} / \mathrm{GHI}$ & $\% \mathrm{GHI} / \mathrm{GHI} *$ & $\begin{array}{r}\% \text { of initial } \\
\text { volume }\end{array}$ & $\begin{array}{r}\% \mathrm{ERL} \\
(\mathrm{GHI} / \mathrm{DHI} / \mathrm{DNI})\end{array}$ & $\% \mathrm{DHI} / \mathrm{GHI}$ & $\begin{array}{r}\% \text { of initial } \\
\text { volume }\end{array}$ & \\
\hline Winster & $0.027 / 0.056 / 1.9$ & 0.14 & 1.0 & 97.0 & $0.054 / 0.044 / 0.078$ & 0.002 & 99.8 & $144621(96.8)$ \\
\hline Palaiseau & $0.0060 / 0.11 / 0.97$ & 0.0090 & 0.28 & 98.7 & $0.0032 / 0.0012 / 0.65$ & 0.0018 & 98.5 & $421458(97.2)$ \\
\hline Payerne & $0.050 / 0.015 / 0.00$ & 0.062 & 0.82 & 93.5 & $0.029 / 0.0039 / 0.00035$ & 0.00 & 98.5 & $262185(92.0)$ \\
\hline Golden & $0.069 / 0.022 / 0.99$ & 0.084 & 0.83 & 98.2 & $0.15 / 0.02 / 0.69$ & 0.00 & 99.2 & $404622(97.3)$ \\
\hline Addu Atoll & $0.033 / 0.61 / 0.57$ & 0.17 & 0.071 & 99.2 & $0.012 / 0.016 / 0.0037$ & 0.00 & 99.9 & 81415 (99.2) \\
\hline Roseraye & $0.14 / 0.41 / 0.093$ & 1.3 & 0.62 & 95.1 & $0.099 / 0.069 / 3.1$ & 0.028 & 94.3 & $133490(92.0)$ \\
\hline
\end{tabular}

Table 4. GHI, DHI and DNI regression slopes from Fig. 2 comparisons together with DHI and DNI slopes found after calibrating for GHI slope.

\begin{tabular}{lrrrrr}
\hline $\begin{array}{l}\text { Linear regression } \\
\text { slopes }\end{array}$ & $\begin{array}{r}\text { GHI } \\
\text { (slope/STDE(\%)) }\end{array}$ & $\begin{array}{r}\text { DHI } \\
(\text { slope/STDE(\%)) }\end{array}$ & $\begin{array}{r}\text { DNI } \\
\text { (slope/STDE(\%)) }\end{array}$ & $\begin{array}{r}\text { DHI after calibration } \\
\text { for GHI slope }\end{array}$ & $\begin{array}{r}\text { DNI after calibration } \\
\text { for GHI slope }\end{array}$ \\
\hline Winster & $1.017 / 4.4$ & $0.966 / 7.8$ & $1.076 / 15.7$ & 0.950 & 1.058 \\
Palaiseau & $0.955 / 4.5$ & $0.901 / 9.1$ & $1.009 / 12.0$ & 0.944 & 1.057 \\
Payerne & $1.002 / 3.7$ & $0.940 / 8.9$ & $1.052 / 10.5$ & 0.937 & 1.049 \\
Golden & $1.021 / 4.2$ & $0.976 / 9.1$ & $1.055 / 8.8$ & 0.956 & 1.033 \\
Addu Atoll & $1.011 / 3.4$ & $0.913 / 9.2$ & $1.062 / 9.5$ & 0.903 & 1.051 \\
Roseraye & $0.993 / 3.8$ & $0.916 / 9.0$ & $1.043 / 11.8$ & 0.922 & 1.050 \\
\hline
\end{tabular}

Table 5. Percentage of data falling within $\pm 20, \pm 40$ and $\pm 60 \mathrm{~W} \mathrm{~m}^{-2}$ in SPN1 - TBM measurement differences (three values at each cell, corresponding to GHI, DHI and DNI irradiances respectively). SPN1 values have been recalibrated as described in Sect. 4.2.

\begin{tabular}{llll}
\hline & P20 & P40 & P60 \\
\hline Winster & $88 / 86 / 70$ & $98 / 98 / 85$ & $100 / 100 / 93$ \\
Palaiseau & $91 / 85 / 64$ & $98 / 98 / 83$ & $99 / 100 / 92$ \\
Payerne & $93 / 85 / 60$ & $99 / 98 / 85$ & $99 / 100 / 94$ \\
Golden & $73 / 87 / 50$ & $96 / 98 / 73$ & $100 / 100 / 86$ \\
Addu Atoll & $76 / 71 / 51$ & $98 / 93 / 82$ & $100 / 98 / 94$ \\
Roseraye & $79 / 75 / 54$ & $97 / 92 / 85$ & $99 / 98 / 94$ \\
\hline
\end{tabular}

conditions. We show in Sect. 5.2.2 that these conditions tend to give the greatest DHI underestimation with SPN1.

Slope values for DNI after correcting SPN1 data for the GHI slope are close to 1.05 for all sites except Golden, which is lower at 1.033. Golden also has the highest average DNI of all the sites (Table 6), which we attribute to its high altitude and continental climate. This means that when the sky is clear, there is little aerosol scatter, and hence low solar aureole values. We show in Sect. 5.2.2 that these conditions tend to give the smallest DNI overestimation. This can be seen in the DNI graph for Golden in Fig. 2, where there is a distinct concentration of high value DNI points close to the $1: 1$ line, and this will reduce the overall slope.

\subsection{Remaining errors after linear recalibration}

In order to remove the above systematic SPN1-TBM differences that were found and make it easier to compare sites, all GHI and DHI SPN1 measurements for each site were recalibrated by dividing by the corresponding regression slopes from Fig. 2 and Table 4. SPN1 DNI was then calculated from the recalibrated DHI and GHI $(\mathrm{DNI}=(\mathrm{GHI}-$ $\mathrm{DHI}) / \cos (\mathrm{SZA}))$. The following sections analyse and look for explanations for the observed remaining differences.

Figure 3 shows the histograms of the remaining differences after recalibration for the three components at the five sites. Table 5 collects the percentage of data falling within $\pm 20, \pm 40$ and $\pm 60 \mathrm{~W} \mathrm{~m}^{-2}$ in the histograms.

Results from Fig. 3 and Table 5 show that the great majority $(>71 \%)$ of GHI and DHI SPN1 measurements agree with TBM within $\pm 20 \mathrm{~W} \mathrm{~m}^{-2}$; the percentage falls for DNI, with only $50 \%$ of the data. More than $96 \%$ and more than $99 \%$ of the SPN1 GHI measurements agree with TBM measurements within \pm 40 and $\pm 60 \mathrm{~W} \mathrm{~m}^{-2}$, respectively. These percentage agreement thresholds fall to 92 and $98 \%$ for DHI and 73 and $86 \%$ for DNI.

Asymmetry is seen in the shape of histograms of DNI errors in Fig. 3. Negative (underestimation) and positive (overestimation) values are related to SPN1 sky sampling for different cloudiness conditions, especially under the presence of bright clouds and their location with respect to the sun. DNI overestimation is related to conditions with bright so- 
Table 6. SPN1 vs. TBM comparison scores before and after slope calibration (three values at each cell, corresponding to GHI, DHI and DNI irradiances respectively).

\begin{tabular}{|c|c|c|c|c|c|c|c|}
\hline & Mean TBM $\left(\mathrm{W} \mathrm{m}^{-2}\right)$ & $\operatorname{MAE}\left(\mathrm{W} \mathrm{m}^{-2}\right)$ & rMAE & $\operatorname{MBE}\left(\mathrm{W} \mathrm{m}^{-2}\right)$ & rMBE & $\operatorname{RMSE}\left(\mathrm{W} \mathrm{m}^{-2}\right)$ & rRMSE $\%$ \\
\hline Winster & $312 / 183 / 215$ & $10 / 11 / 30$ & $3.3 / 5.9 / 14$ & $3.8 /-7.6 / 30$ & $1.2 /-4.2 / 14$ & $15 / 16 / 44$ & $4.9 / 8.8 / 21$ \\
\hline Palaiseau & $318 / 160 / 281$ & $16 / 18 / 22$ & $4.9 / 11 / 7.8$ & $-14 /-17 / 14$ & $-4.4 /-11 / 4.8$ & $23 / 24 / 34$ & $7.3 / 15 / 12$ \\
\hline Payerne & $358 / 160 / 314$ & $8.3 / 13 / 28$ & $2.3 / 8 / 8.9$ & $0.77 /-11 / 27$ & $0.21 /-7 / 8.6$ & $13 / 18 / 41$ & $3.8 / 12 / 13$ \\
\hline Golden & $471 / 154 / 514$ & $18 / 10 / 44$ & $3.7 / 6.6 / 8.5$ & $11 /-4.6 / 41$ & $2.4 /-3 / 8$ & $23 / 15 / 58$ & $4.9 / 9.6 / 11$ \\
\hline Addu Atoll & $532 / 221 / 426$ & $15 / 23 / 42$ & $2.8 / 11 / 9.8$ & $4.6 /-22 / 41$ & $0.86 /-10 / 9.6$ & $19 / 30 / 53$ & $3.6 / 14 / 12$ \\
\hline Roseraye & $467 / 231 / 326$ & $13 / 21 / 31$ & $2.8 / 9.2 / 9.4$ & $-2.7 /-19 / 29$ & $-0.58 /-8.4 / 8.9$ & $18 / 31 / 44$ & $3.9 / 13 / 14$ \\
\hline
\end{tabular}

SPN1 values after recalibration as described in Sect. 4.2.

\begin{tabular}{|c|c|c|c|c|c|c|c|}
\hline & Mean TBM $\left(\mathrm{W} \mathrm{m}^{-2}\right)$ & $\operatorname{MAE}\left(\mathrm{W} \mathrm{m}^{2}\right)$ & rMAE & $\left(\mathrm{W} \mathrm{m}^{-2}\right)$ & rMBE & $\operatorname{RMSE}\left(\mathrm{W} \mathrm{m}^{-2}\right)$ & rRMSE \% \\
\hline Winster & $312 / 183 / 215$ & $9.3 / 10 / 19$ & $3 / 5.5 / 9.0$ & $-1.4 /-1.4 / 8.9$ & $-0.44 /-0.77 / 4.1$ & $14 / 15 / 30$ & $4.4 / 8 / 14$ \\
\hline Palaiseau & $318 / 160 / 281$ & $8.8 / 11 / 22$ & $2.8 / 6.7 / 7.8$ & $0.31 /-1.7 / 10$ & $0.098 /-1.1 / 3.6$ & $15 / 16 / 34$ & $4.7 / 10 / 12$ \\
\hline Payerne & $358 / 160 / 314$ & $8.2 / 11 / 22$ & $2.3 / 6.6 / 7$ & $-0.023 /-1.5 / 7.8$ & $-0.0063 /-0.97 / 2.5$ & $13 / 15 / 31$ & $3.7 / 9.5 / 9.8$ \\
\hline Golden & $471 / 154 / 514$ & $15 / 10 / 30$ & $3.1 / 6.5 / 5.8$ & $1.1 /-0.92 / 18$ & $0.23 /-0.6 / 3.4$ & $19 / 14 / 42$ & $4.1 / 9.3 / 8.1$ \\
\hline Addu Atoll & $532 / 221 / 426$ & $14 / 16 / 25$ & $2.6 / 7.2 / 5.8$ & $-1.1 /-3.3 / 4.9$ & $-0.2 /-1.5 / 1.1$ & $18 / 22 / 32$ & $3.3 / 10 / 7.6$ \\
\hline Roseraye & $467 / 231 / 326$ & $13 / 15 / 24$ & $2.7 / 6.6 / 7.3$ & $0.46 /-0.038 / 4.8$ & $0.097 /-0.016 / 1.5$ & $18 / 23 / 33$ & $3.9 / 9.8 / 10$ \\
\hline
\end{tabular}

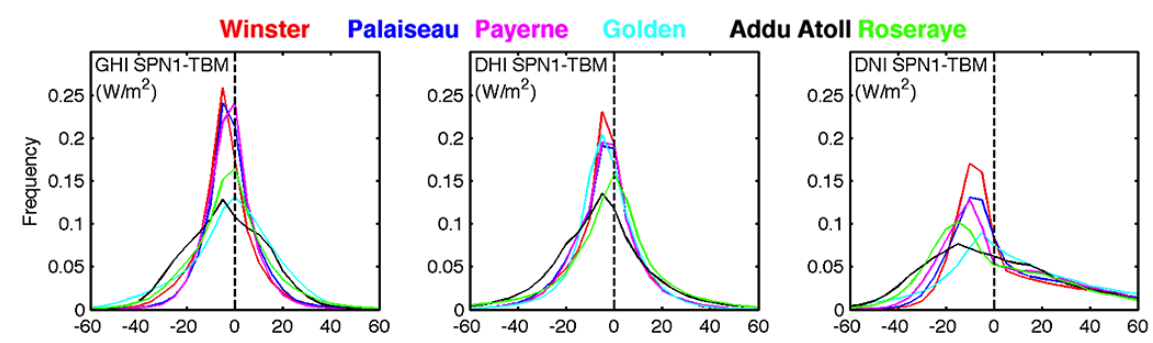

Figure 3. SPN1 - TBM difference distributions (after slope calibration) for GHI (left), DHI (centre) and DNI (right) irradiance at all sites.

lar aureole (circumsolar radiation) and DNI underestimation is related to bright clouds far from the sun position in the sky (DHI overestimation). The asymmetry seen in the DNI histograms is related to the proportion of time during which these conditions are present. This will be discussed in more detail in the next section.

Table 6 collects the SPN1 vs. TBM metrics results for the indicators presented in Sect. 3 (MAE, MBE and RMSE) in absolute and relative (to the mean TBM) values. Two tables are presented: one for the case where SPN1 data are considered without applying the linear slope recalibration factors and the other with this recalibration applied as described above.

As expected, all error metrics get closer to 0 , in particular MBE and rMBE, after the recalibration factors are applied. rMAE values after recalibration are generally consistent among the different sites: for GHI (rMAE values are between 2.3 and $3.1 \%$ ) and DHI (6.5-7.2\%, except for Winster, $5.5 \%)$ and DNI (7.3-9.0\%, 5.8\% for Golden and Addu Atoll).

rMBE gets small $(|\mathrm{rMBE}|<1.5 \%)$ for GHI and DHI as expected after having applied slope recalibration factors.
Larger and positive biases are found for DNI, of 1.1-4.1, being lower for the two inter-tropical sites.

Notice that mean TBM DHI values are larger for the two tropical sites (221-231 $\mathrm{W} \mathrm{m}^{-2}$ ) than for the other four (154$183 \mathrm{~W} \mathrm{~m}^{-2}$ ). Golden has the lowest mean DHI, and also the highest mean DNI, so is the sunniest site.

\section{Uncertainty sources}

In this section the differences found between SPN1 and TBM measurements are analysed in detail in order to identify and describe the sources of uncertainties that are affecting the comparison. For most of these analyses, the recalibrated (see Sect. 4.1) SPN1 measurements are used. Two main categories of uncertainties have been listed depending on their origin: relating to the solar direct beam, and to the diffuse and circumsolar radiation. 

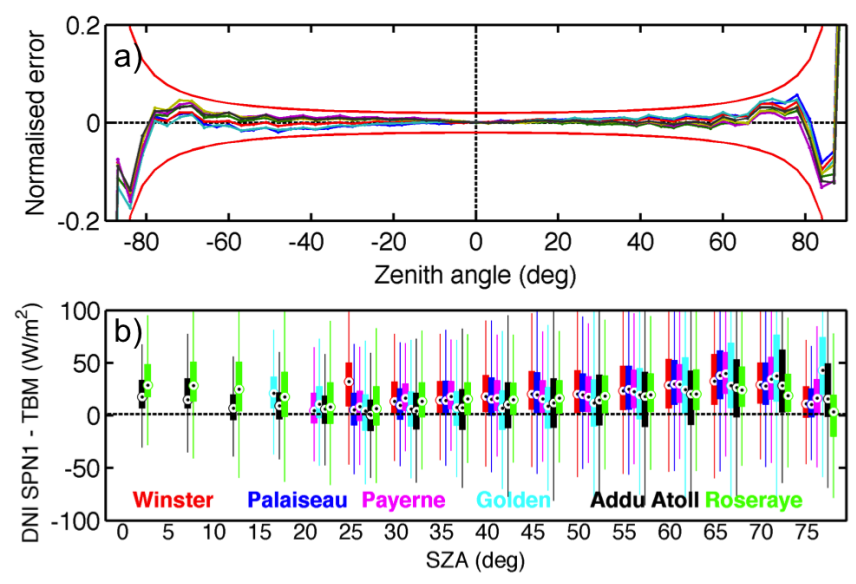

Figure 4. (a) Cosine response from laboratory tests. Shown error is normalized by incoming beam intensity. Thermopile readings are shown in different colours. Red envelope lines show the $\pm 2 \%$ limits (from ISO 9060/1990). (b) SPN1 - TBM differences distributions for DNI, GHI and DHI as a function of bins of SZA at all stations. Box size represents the percentiles 25 and 75 . Whiskers account for $99.3 \%$ of data under Gaussian distribution assumption.

\subsection{Direct beam uncertainties}

\subsubsection{Cosine response}

One of the key factors affecting the direct beam response is the cosine response. Laboratory tests show a generally flat response out to around $65^{\circ}$ of SZA, then a small rise, with the response falling off after $80^{\circ}$ (see Fig. 4a). The graph also shows the $\pm 2 \%$ limits (from ISO 9060/1990, WMO, 2010), and an indication of the variability between the seven detectors within an instrument.

Figure $4 \mathrm{~b}$ shows the DNI SPN1 - TBM differences as a function of SZA for all the sites. Results for DNI confirm the laboratory tests for the general flat response and a small rise around $65^{\circ}$, which is due to the geometry shape of the thermopile diffusers.

However, an increase in DNI SPN1 - TBM differences is found for very low SZA below $15^{\circ}$, which does not follow the laboratory measurements. This may be because for $\mathrm{SZA}<15^{\circ}$, the effective opening angle (see Sect. 5.2.3) is always large, whereas for larger SZA, the opening angle varies from large to small with SAA. This means that for very low SZA, the DNI always includes a large part of the solar aureole, whereas for larger SZA the aureole contribution is smaller when averaged over the range of SAA.

\subsubsection{Detector matching, dome lensing effect, detector hopping}

The operation of the SPN1 relies on close matching of all the detectors to give a smooth output over the day, as different detectors become exposed or shaded. The graphs in Fig. 5b

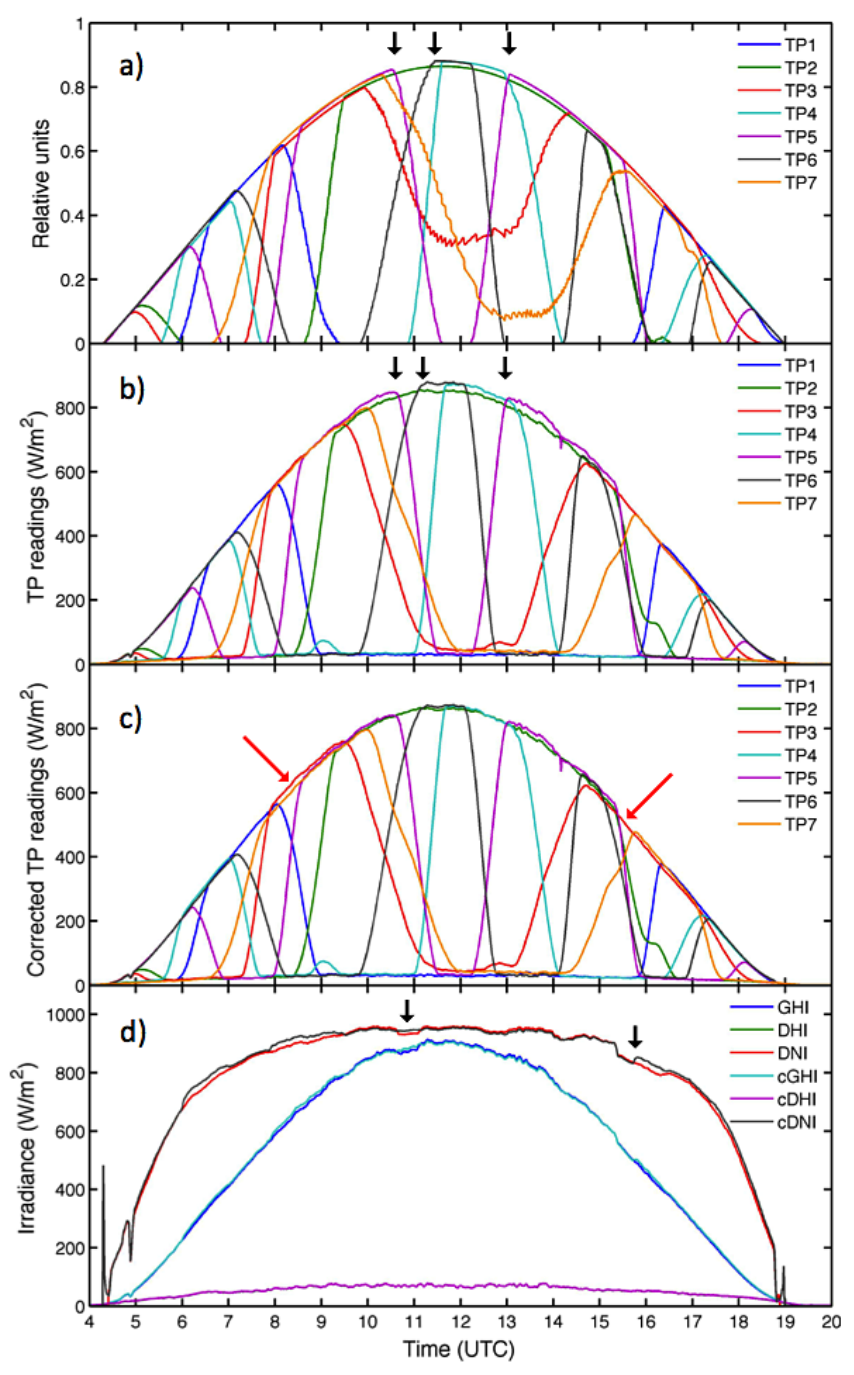

Figure 5. (a) Theoretical model of reading outputs from each of the SPN1 7 individual thermopiles (TPs), including dome lensing, for 1 August 2013 at Payerne. (b) Actual SPN1 TP readings for this same day and place on a clear-sky day. (c) TP readings after correction for dome lensing. (d) Measured and corrected GHI, DHI and DNI measurements for this clear-sky day.

show how the outputs from the individual detectors rise and fall throughout the day as they are exposed to or shaded from the sun. The GHI output will follow the upper bound plus the lower bound of these measurements, and the DHI output will follow twice the lower bound (see Eq. 3). This means that there may be apparent step changes, particularly in the GHI output trace, if different detectors are mismatched. There are two identified reasons for this:

1. Dome lensing effect. Because six of the seven detectors are located away from the centre of the protective glass dome, light reaching them from a point source is bent asymmetrically as it passes through the dome. This has the effect of increasing the measured irradiance on de- 
tectors that are on the same side of the SPN1 as the light source, and reducing the measured irradiance on detectors that are on the opposite side. This effect is calculable and predictable from the known position of the sun, provided that the SPN1 orientation is known.

2. Detector mismatches. There are several other possible sources of mismatch, such as incorrect detector calibration, imperfect horizontal mounting of the diffuser, or other manufacturing variations, which may cause detector mismatches for different solar positions. These are generally not predictable in advance.

Figure 5a shows the calculated outputs for "perfect" detectors through the course of a day in Payerne in response to a uniform point source of light, taking account of the effect of dome lensing. The arrowed points show transitions between detectors on the near and far sides of the SPN1 relative to the sun, which will result in steps in the GHI output trace at these points.

Figure $5 \mathrm{~b}$ shows the actual detector outputs for the same day, which was cloud-free. The lower bound of the detector traces (MIN values) is raised due to the small amount of diffuse radiation on this day. Close inspection shows that the upper values for the fully exposed traces (MAX values) agree well with the modelled ones. Again, small steps in the GHI output would be visible corresponding to the arrowed transitions.

Figure $5 \mathrm{c}$ shows how the individual detector traces would look after correcting the actual outputs for the effects of dome lensing. This results in a tighter clustering of the exposed detector readings, which should reduce the variability in the GHI output trace. The main exception is the red trace (TP3, in red, marked by arrows), which now shows a higher value in the morning, and a lower value in the afternoon, than the other exposed detector traces. Our interpretation is that this diffuser is mounted with a small deviation angle with respect to the horizontal, sloping down towards the east in this case.

Figure 5d shows the resulting SPN1 GHI, DHI and DNI curves for this day, for both the original and corrected individual detector readings. It can be seen that the step near 11:00 UTC is well corrected, and that the step near 16:00 UTC is partly removed, though there is still a step remaining here due to the asymmetric response of TP3.

The dome lensing effects are typically of the order of up to $\pm 15 \mathrm{~W} \mathrm{~m}^{-2}$ in the horizontal irradiance readings in bright sunshine. Steps due to detector mismatch are typically of a similar size. The two effects can add together and make larger steps like those observed in Fig. 5.

\subsection{Diffuse uncertainties}

While the GHI output of the SPN1 is generally a close equivalent of a pyranometer measurement (as discussed in Sect. 4), the DHI differences are more important in terms of relative values though similar in absolute values. There are two main contributions to this:

1. the large spectral differences in diffuse light between clear blue sky and overcast conditions;

2. non-isotropic diffuse radiance distribution, particularly in blue-sky or bright cloudy conditions. The DHI measurement is based on the lowest of the seven detector readings, each of which sees a different $50 \%$ sample of the sky hemisphere. In cases where more than one sensor is fully shaded from the sun, the SPN1's algorithm will always use the sample with the lowest measured value for calculating the DHI. This results in a negative bias compared to the TBM.

These two effects are discussed in the following subsections.

\subsubsection{Spectral response}

The spectral response of the SPN1 falls steeply at the blue end of the spectrum, as shown in Fig. 6. In the near infra-red (NIR) part, SPN1 has a higher sensitivity compared to the TBM. These spectral effects can be particularly noticeable in the measurements under two conditions.

1. The DHI output under clear blue skies is typically much lower than the TBM, due to the predominantly blue weighting of the diffuse blue sky spectrum.

2. Under heavy overcast conditions both GHI and DHI outputs are low compared with the TBM (they are also approximately equal because there is no direct beam). This appears to be because the NIR part of the solar spectrum (where the SPN1 spectral response is higher) is preferentially absorbed by optically thick cloud.

To isolate the effects of spectral response from any other effects, a modified SPN1 with no internal shadow mask was used on the Winster sun-tracker, shaded by a $\pm 2.5^{\circ}$ shading disc (during the same period as in Table 2). The DHI measurements from this modified SPN1 compared to the TBM DHI are presented in Fig. 6 with the colour code representing data sorted in 0.1 width bins with respect to the cloud fraction from 0 to 1 . The dark blue points correspond to clear sky conditions, and show a low sensitivity to diffuse light from blue sky. As the cloud fraction increases (green-yellow points), the SPN1 diffuse sensitivity increases, approaching perfect agreement (1:1 line) for cloud fractions near 1 (red points, corresponding to cloudy sky). More precisely, fitting linear regressions for each $\mathrm{CF}$ shows a progressively increasing slope with $\mathrm{CF}$, from 0.72 for $\mathrm{CF}=0$ to 0.93 for $\mathrm{CF}=1$. There is already a simple correction for this effect implemented in the commercial SPN1 firmware, so the normal measured differences are much less than shown in this graph (as has been seen in Fig. 2 and Table 4). The firmware correction increases the measured DHI by a factor of 1.14 , which corrects for typical mixed-cloud conditions. 

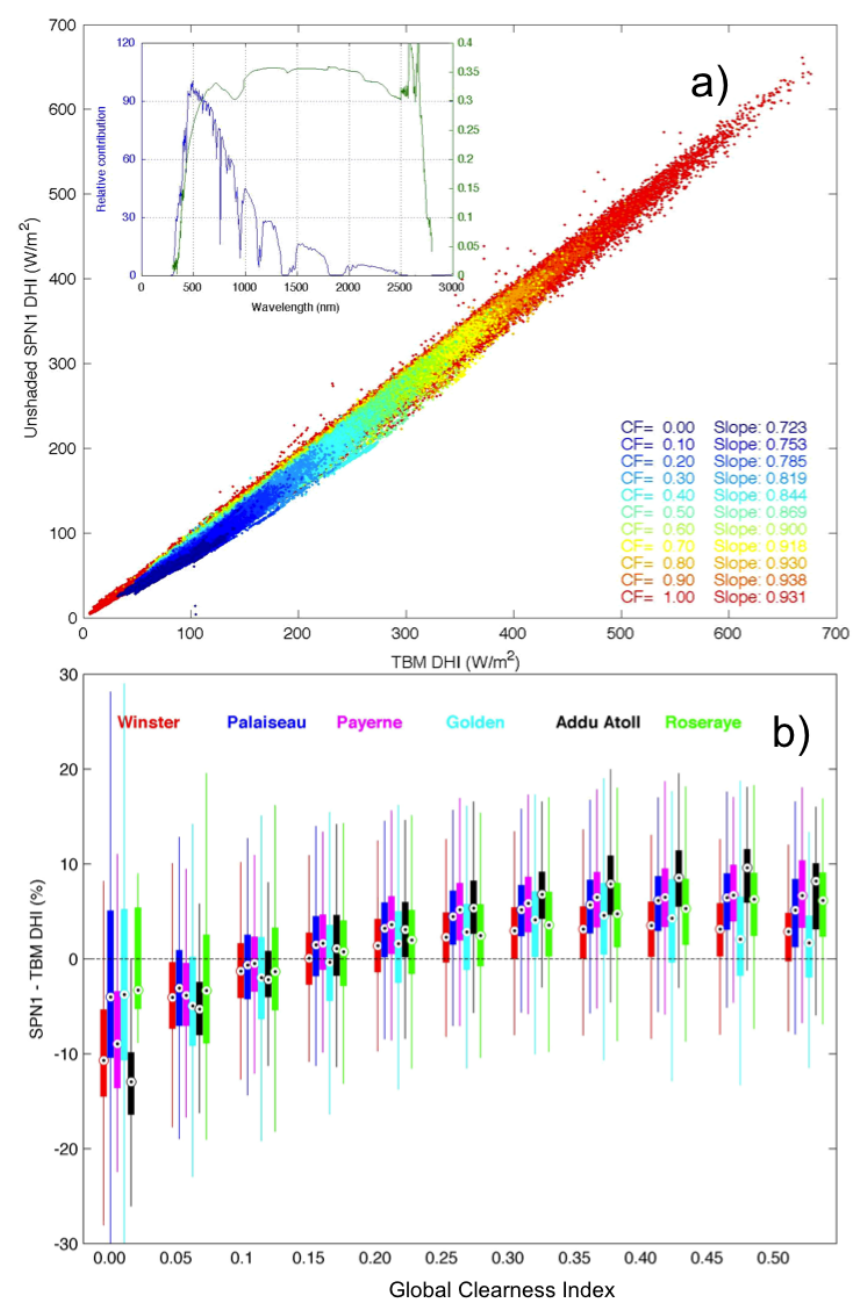

Figure 6. (a) Inner plot: Standard solar spectrum (AM 1.5) and SPN1 spectral response. Main plot: SPN1 (with no internal shadow mask) DHI vs. TBM DHI sorted by cloud fraction bins (in colour scale) at Winster. The regression slope values for each bin are shown on the bottom right corner. No calibration was applied to SPN1 data. (b) DHI differences vs. clearness index in box plots under suncovered conditions (GHI equals DHI within $3 \%$ ) at all sites. SPN1 values have been recalibrated as described in Sect. 4.2. Box size represents the percentiles 25 and 75 . Whiskers account for $99.3 \%$ of data under Gaussian distribution assumption.

The heavy overcast conditions can be clearly seen by selecting readings when $\mathrm{GHI}=\mathrm{DHI}$ (with $3 \%$ of tolerance), and plotting the percentage difference between SPN1 and TBM against global clearness index defined as the ratio of measured GHI to GHI at the top of the atmosphere (TOA). This is shown in Fig. $6 \mathrm{~b}$.

For very low clearness index values when the clouds are thick, the SPN1 sensitivity reduces as clearness index reduces. Note that this may also include periods when the SPN1 and TBM instrument domes are covered with water droplets, which strongly absorb the NIR part of the solar spectrum. Values of clearness index greater than 0.3 are typical of lighter cloud, with a diffuse spectrum very similar to global.

\subsubsection{Direct/Diffuse partition}

Considering the sky radiance, there is always a zone close to the sun where the radiance reduces with distance from the sun, from that of the bright solar disc towards the average brightness of the diffuse sky. The shape of this curve depends largely on the aerosol and water content of the atmosphere, with liquid water or ice having a large effect. Gueymard (2001) shows some good examples of this variation for different atmospheric constituents, and SegalRosenheimer (2013) describes some modelling of the effect of cirrus clouds on the solar aureole (Segal-Rosenheimer et al., 2013).

The cut-off point at which the radiance is counted as DNI or DHI is to some extent arbitrary, and is usually made at $2.5^{\circ}$ away from the sun based on the opening half-angle for pyrheliometers recommended by the CIMO guide (WMO, 2010).

As mentioned above, the DNI measurement from SPN1 is derived from the difference between the highest reading of the seven thermopile detectors (MAX), which is fully exposed to the sun, and the lowest reading (MIN), which is fully shaded from the sun. Where more than one detector is exposed to the sun, the highest reading is always used. The effective aperture for this measurement changes with the relative position of the sun with respect to SPN1 shadow mask, but it is always at least $\pm 5^{\circ}$, which is significantly larger than a standard pyrheliometer $\left( \pm 2.5^{\circ}\right)$ (WMO, 2010). This means that DNI measurement from SPN1 will include a larger part of the circumsolar aureole compared to standard pyrheliometers. In other words, in most cases the DHI measurement will exclude a part of the aureole energy contribution, thus leading to DHI underestimation and DNI overestimation from SPN1 when compared to a standard TBM.

\section{Solar aureole measurement}

There are few reported measurements of the intensity of the solar aureole with respect to the considered opening halfangle. For example, Wilbert et al. $(2011,2012)$ reported aureole intensity measurements for angles between 0.475 and $5^{\circ}$ from the centre of the sun disc. To understand the aureole for larger opening half angles relevant to the SPN1, the Winster tracker was operated with an additional shaded pyranometer with a larger shading disc covering $\pm 6.5^{\circ}$ (referred to as $\mathrm{DHI}_{6.5}$ ) during the same period as the reference TBM measurements made using a $2.5^{\circ}$ shading ball (referred to as $\mathrm{DHI}_{2.5}$ in this section). The difference between these two measurements is the circumsolar horizontal irradiance between the half angles 2.5 and $6.5^{\circ}$, referred to as aureole energy in this paper. 

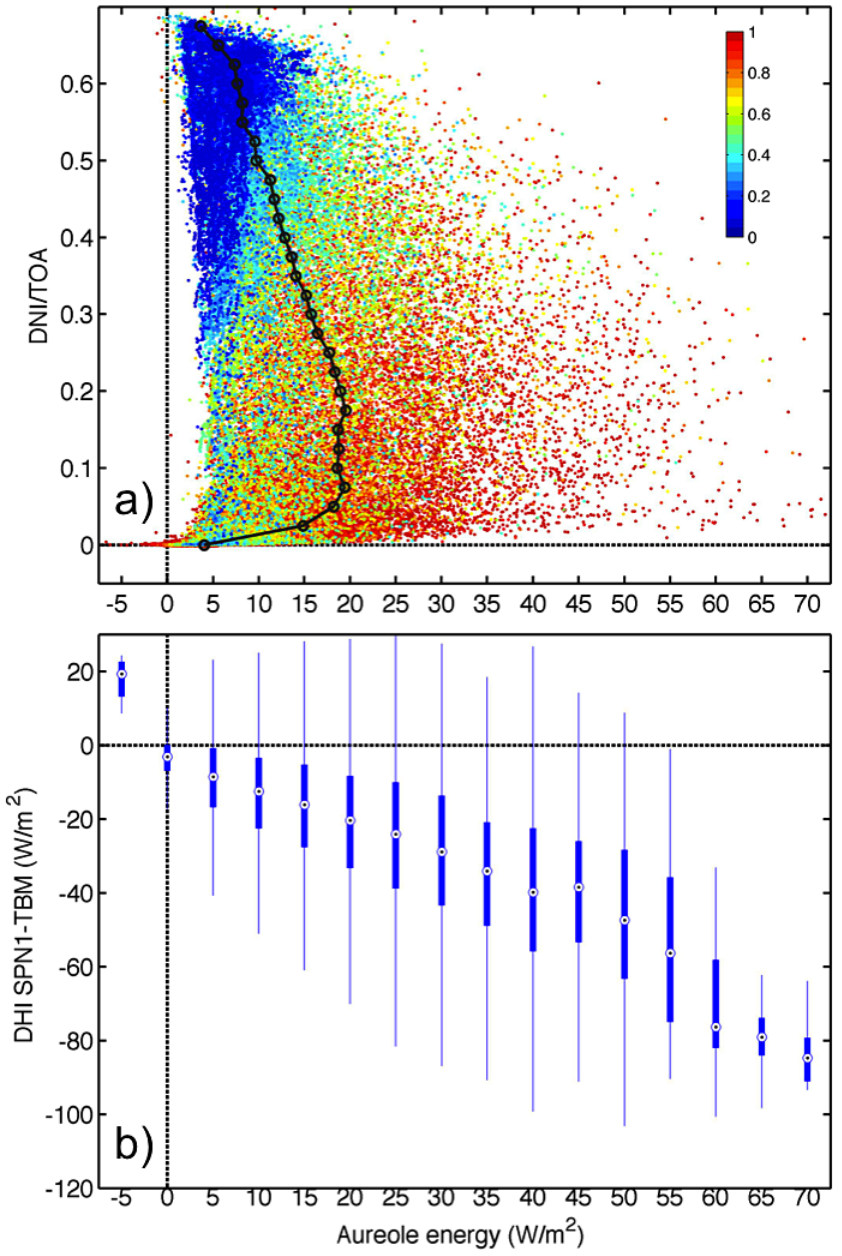

Figure 7. (a) DNI/TOA vs. aureole energy; colour scale corresponds to $\mathrm{CF}(\mathrm{CF}=0$ in blue, $\mathrm{CF}=1$ in red). Black line represents the mean aureole values for each 0.025 -wide bin of DNI/TOA. (b) DHI SPN1 - TBM differences in box plots as a function of aureole energy at Winster. Box sizes account for 25-75 percentile interval. Whisker length corresponds to approximately $\pm 2.7 \sigma$ and $99.3 \%$ coverage if the data are normally distributed.

The solar aureole energy is low for both pristine clear-sky and heavy overcast conditions and it tends to be larger as aerosol load increases, which increases the amount of forward scattering in the vicinity of the sun. It also increases for cloudy conditions when thin clouds of cirrus type are partly covering the sun or when there are surrounding broken clouds, in which case the aureole brightness can be both large and variable over short timescales. It should also be noted that the energy in the part of the aureole between the edge of the solar disc at 0.266 and $2.5^{\circ}$ will be of a similar size to that between 2.5 and $6.5^{\circ}$.

Figure 7a shows the direct clearness index defined as the ratio of measured DNI to DNI at the TOA, plotted against the aureole energy. Again, the colour scale represents the CF. The mean aureole energy for each direct clearness index bin is also plotted. This shows that for clear skies, there is little forward scattered radiation, and the aureole energy is low. As the aureole energy increases, because the forward scattering around the sun increases, the direct clearness index tends to reduce and CF increases. For very low clearness index (with opaque clouds obscuring the sun), there is little remaining direct beam, and the aureole tends to reduce back to zero. Some exceptions to this are seen in Fig. 7a, with low direct clearness index but large aureole energy, coming from scattered light around the sun that passes through the clouds under anisotropic conditions. Large aureole is related to large $\mathrm{CF}$ while low aureole intensity can be related to all cloudy conditions.

\section{SPN1 dependence on aureole intensity}

Figure $7 \mathrm{~b}$ shows the SPN1-TBM DHI 2.5 differences at Winster as a function of the aureole energy. It shows that the DHI underestimation by SPN1 increases with solar aureole energy, with a mean relationship close to $-1 \mathrm{~W} \mathrm{~m}^{-2}$ for each $1 \mathrm{~W} \mathrm{~m}^{-2}$ of aureole energy.

Table 7 compares the resulting DHI linear regression slope (after calibrating for the GHI slope, as in Table 4) and standard deviation values (analogous to the ones for Fig. 2) when using $\mathrm{DHI}_{2.5}$ and $\mathrm{DHI}_{6.5}$ over the whole measurement period at Winster. The slope is much closer to 1 and the standard deviation is reduced by $12 \%$ for the $6.5^{\circ}$ shading disc, showing that a large part of the differences observed with the DHI and hence also DNI from the SPN1 compared to TBM is explained by the different opening angles of the two types of instruments.

\subsubsection{Sky sampling, effective aperture change and aureole effects}

As mentioned in the previous section, the effective aperture for SPN1 changes with the relative position of the sun with respect to SPN1 shadow mask. This is due to its intrinsic design that, for example, leads to larger aperture angles when the detector with the lowest of the seven readings is just behind one "leg" of the shadow mask, which happens, for SZA $>20^{\circ}$, with $60^{\circ}$ of azimuth periodicity (see Fig. 1). This effect can be easily observed for measurements at mid-latitude sites with the DHI differences plotted against the SAA, as shown in Fig. 8 using the data from Winster, Palaiseau, Payerne and Golden.

Figure 8a shows the DHI differences vs. SAA with box plots for four different bins of cloud fractions $(0-0.1,0.1$ $0.5,0.5-0.9,0.9-1)$.

For Palaiseau, systematic DHI underestimation from SPN1 of about $-20 \mathrm{~W} \mathrm{~m}^{-2}$ are observed for SAA around 120,180 and $240^{\circ}$ of SAA, showing a $60^{\circ}$ periodicity. Conversely, DHI differences are close to zero for SAA around $90,150,210$ and $270^{\circ}$ of SAA. In Palaiseau, SPN1 is oriented in such a way that the main axis of the shadow mask is 


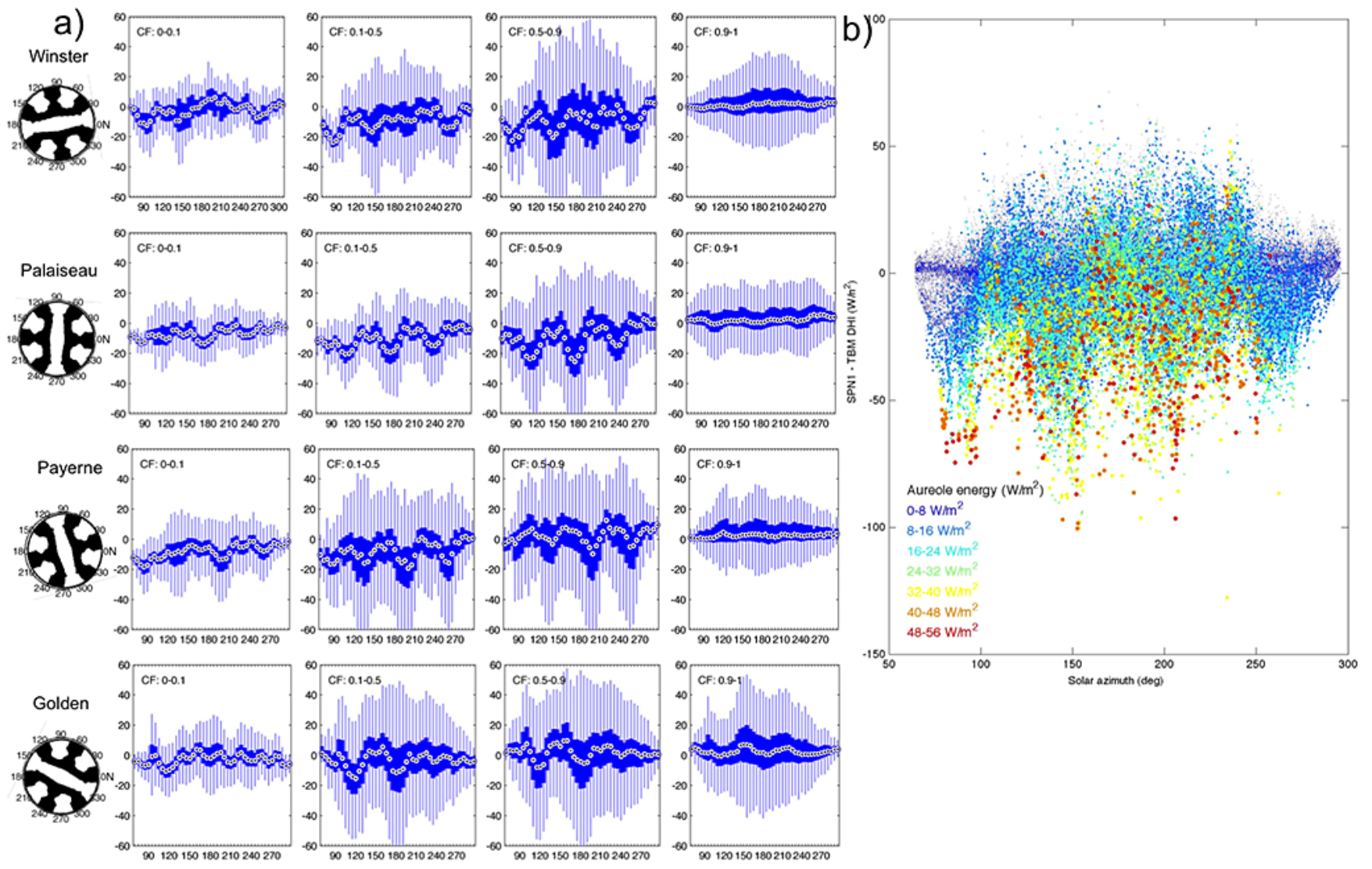

Figure 8. DHI differences (in $\mathrm{W} \mathrm{m}^{-2}$ ) as a function of solar azimuth angle (in degrees) and for different (a) cloud fraction values at Winster, Palaiseau, Payerne and Golden and (b) aureole intensities at Winster. The SPN1 shadow mask orientation with respect to solar azimuth $\left(0^{\circ}=N\right)$ is shown for each site (circle drawings on the left). SPN1 values have been recalibrated as described in Sect. 4.2.

oriented East-West (see Table 1), so that the mask's legs are pointing to $0,60,120^{\circ}$, etc. For Payerne, although the SPN1 is oriented with a difference of -161 degrees compared to Palaiseau, the observed effect is consistent, with only a $19^{\circ}$ shift in SAA corresponding to $-161^{\circ}$ modulo $180^{\circ}$.

For Winster and Golden, compared to Palaiseau, the SPN1 are oriented with a difference of respectively 90 and $60^{\circ}$. One can observe the same type of patterns in the DHI differences with the corresponding shift in SAA, but this effect seems to be less clear than for Palaiseau.

This SAA effect of DHI periodic underestimation is less significant for overcast and very clear-sky conditions, for which the sun aureole energy is smaller, and the diffuse sky radiance more isotropic. The largest effect is in fact observed for partly cloudy conditions; that is, when it is also more likely that diffuse irradiance is highly anisotropic, in particular for cloud fraction between 0.5 and 0.9 .

Figure $8 \mathrm{~b}$ shows, for Winster, these azimuthal dependencies of DHI differences in a scatter plot with the points colour scaled for measured aureole intensity as in the previous section (that is, $\mathrm{DHI}_{2.5}-\mathrm{DHI}_{6.5}$ ). As a conclusion, the strength
Table 7. Slopes of regression line and standard deviation of the Winster TBM vs. SPN1 DHI measurements for different opening angles. Recalibration for GHI slope applied to all readings, as in Table 4.

\begin{tabular}{lll}
\hline Tracker shading disc & $\mathrm{TBM} \pm 2.5^{\circ}$ disc & $\mathrm{TBM} \pm 6.5^{\circ}$ disc \\
\hline DHI slope & 0.953 & 0.98 \\
DHI SD $(\%)$ & 4.3 & 3.8 \\
\hline
\end{tabular}

of the periodic variation of the DHI errors with respect to the SAA also depends on the intensity of the solar aureole.

For the case of low sun aureole energy, DHI differences are close to 0 and the SAA dependency is nearly flat. As the aureole becomes brighter, DHI differences are becoming more negative (as already seen for Fig. 7). Large underestimations are observed for cases with high aureole energy (orange and red points) following the expected $60^{\circ}$ periodic pattern.

There are similar patterns for the tropical sites, but here the main variation is with solar zenith angle. 

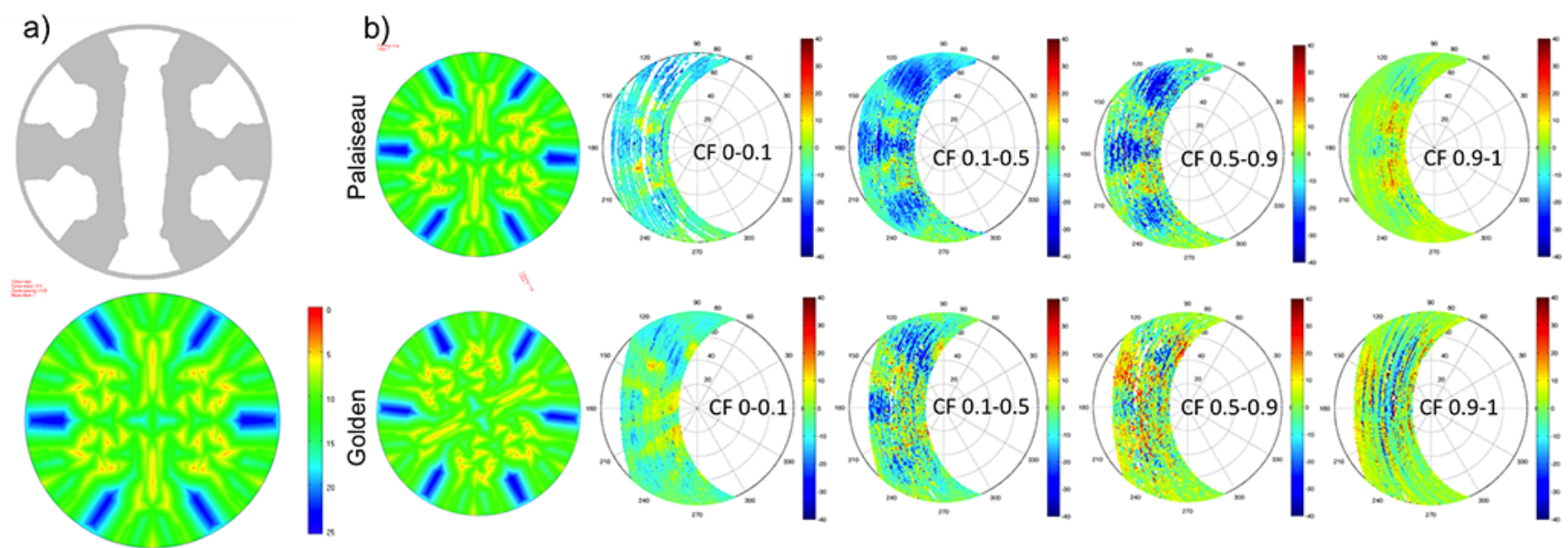

Figure 9. (a) Modelled diffuse first touch angles for the SPN1 relative to the shadow mask. Colour scale gives angle in degrees. (b) DHI differences (TBM - SPN1) in polar coordinates (of SZA and SAA) at Palaiseau and Golden for different bins of CF with the first touch angles pattern rotated for easier visual comparison.

Actually, we find that the diffuse errors are sensitive to both azimuth and zenith solar angles, and a more sophisticated analysis is presented next to grasp the full picture.

\section{Modelling of SPN1 effective aperture}

The SPN1's thermopiles and its shadow mask arrangement have been modelled in 3-D to assess the effective opening aperture of the SPN1 for different solar positions. To simplify calculations, and because the actual sample of sky seen by each sensor is always asymmetric around the sun, the size of the largest complete circle around the sun that is seen as fully exposed or fully shaded is calculated. We have called this the "first touch angle", being the point at which a circle of increasing radius around the sun first touches an edge of the SPN1 shading pattern as seen by a specific detector. This gives a lower limit on the effective aperture angle of the SPN1 for this solar position. For the diffuse measurements, these are likely to be taken from the thermopile that has the largest first touch angle, and hence includes the smallest amount of solar aureole. The diffuse first touch angle is therefore the value of the largest first touch angle from the group of shaded sensors for any given solar position. Further explanations about the first touch angle definition can be found in the Supplement.

The diffuse first touch angles are plotted in Fig. 9a by colour value for all possible solar positions relative to the SPN1 orientation (in polar coordinates of SZA and SAA). The image colour map shows low angles in red, and higher angles in blue, to match the expected diffuse differences. The red/orange areas represent solar positions where the first touch angle is as low as $5^{\circ}$, and the blue areas represent first touch angles greater than $20^{\circ}$. From this analysis we can see that the effective aperture for the SPN1 is always more than $\pm 5^{\circ}$, with a maximum value of $\pm 25^{\circ}$. The SPN1 will there- fore exclude a large part of the solar aureole from its diffuse measurement (and include it in the DNI measurement), but the amount that is excluded will vary with solar position.

In Fig. 9b the diffuse differences for Palaiseau and Golden are plotted on the same polar projection, with the first touch angle plot rotated to match the SPN1 orientation. A correlation can be observed between the varying effective opening angle of the SPN1, and the measured diffuse differences. The plots are shown separated out according to the cloud fraction. There is a clear correlation between the larger diffuse first touch angles and the amplitude of the negative diffuse differences (blue regions match). This is obvious for all conditions, but is most strongly seen for intermediate values of cloud fraction. For sky conditions close to overcast (CF from 0.9 and 1) DHI differences show a similar pattern as for cloudless-sky conditions ( $\mathrm{CF}$ from 0 to 0.1 ), with less underestimation for the former case, due to the spectral effects discussed in Fig. 6a. However, at Golden, the DHI differences pattern, which is well defined for cloudless conditions, disappears for overcast conditions.

In Fig. 10, the DHI SPN1 - TBM differences are plotted against SZA and SAA for all sites using the same polar projection and including all cloud conditions. The first touch angle diagram of Fig. 9a is simplified by plotting only the isolines for $10^{\circ}$, and these contours are superimposed on the plots in the correct orientation. From this figure, it can be seen that the correlation is obvious for all sites. This correlation is more significant where the data set is larger (Golden, Palaiseau and Payerne), where the large variability of the diffuse sky in mixed cloud conditions is averaged out over time. The correlation will also depend on the cloud regime, and will be most clearly seen in conditions of either high turbidity in clear skies, or light clouds when overcast. 


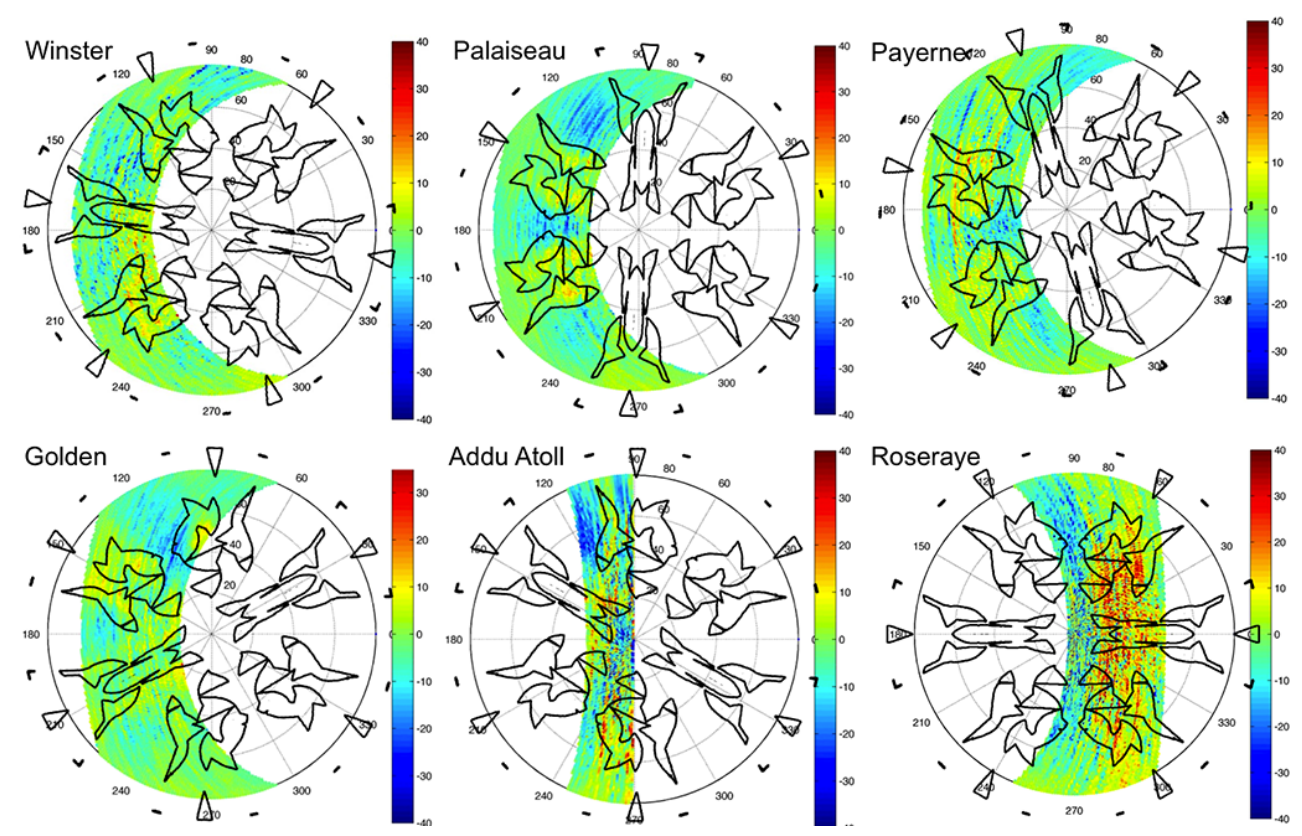

Figure 10. DHI differences (TBM - SPN1) as in Fig. 9 with the $10^{\circ}$ first-touch-angle isolines superimposed for all sites. SPN1 values have been recalibrated as described in Sect. 4.2.

Modelling of these aureole effects on the direct beam may be possible, but is complicated as DNI is retrieved from two thermopile measurements (one measuring MAX and the other measuring MIN) so it combines the different aureole views from at least two detectors. The DNI errors of SPN1 are dominated by the dome lensing and detector mismatch effects (Sect. 5.1.2), so the aureole effects are more difficult to distinguish.

\section{Conclusions}

The SPN1 is proving popular for use on solar measurement sites due to its ruggedness and simplicity of operation.

We have shown that the SPN1 performance is consistent across all of the sites we have studied, and have attempted to describe the main sources of difference between the SPN1 and tracker-based measurements. The most important ones are related to the DHI measurement, mainly due to spectral and sky sampling issues. This means that, when GHI and DHI are recalibrated, MAE is around $3 \%$ or better and it is 2-3 times larger for DHI and DNI.

Understanding these differences will help inform future data analysis and correction algorithms, as well as instrument design.

\section{Further work}

This analysis suggests several possibilities for improving SPN1 accuracy and agreement with tracker-based measure- ments, which we hope to present in a future paper. The main proposed approaches to this are as follows:

- on-site recalibration of the SPN1

- once SPN1 is well calibrated for GHI, testing for a simple extra factor of 1.05 for DHI, and 0.95 for DNI readings for mid-latitude sites, as a simple solution suggested by results in Table 4

- further correction of DHI and DNI measurements, which could be based on the diffuse ratio (SPN1 DHI / GHI, as a proxy for cloud fraction), the clearness index, or/and on estimations of solar aureole intensity (that is, the circumsolar horizontal irradiance) and cloud fraction from other measurements

- correction of dome lensing and detector matching errors on the DNI measurement using outputs of the individual detectors. The SPN1 orientation would be needed for that, and it could be retrieved offline from individual thermopile measurements and information about the site location

- correction of the DHI measurements using outputs of the individual thermopile and knowledge of SPN1 orientation, along with solar aureole and cloud fraction estimations. 


\section{Supplement}

Additional supporting information may be found in the online version of this paper.

Section S1: Three case study examples of SPN1 and TBM; GHI, DHI and DNI diurnal cycle comparison for Palaiseau together with TSI images for these days. One figure illustrates each case. Fig. S1: A cloudless day with large aureole energy due to important aerosol load (AOD at $500 \mathrm{~nm}$ of 0.2 in the morning and increasing to 0.3 in the afternoon). This is a good example of the azimuth effect seen in Fig. 8. Figure $\mathrm{S} 2$ : The same effect (but larger) is seen in this case caused by the presence of bright clouds around the sun. Figure S3: Day with mainly broken clouds passing (and cirrus appearing at the end of the afternoon) that lead to moments with almost cloudless, sky heterogeneous radiance and nearly overcast conditions. Consequently, quite diverse DHI SPN1-TBM difference values are found, from -50 to $+50 \mathrm{~W} \mathrm{~m}^{-2}$.
Section S2: Polar plots for GHI and DNI SPN1 - TBM. Figures S4 and S5: Same as Fig. 10 but for GHI and DNI SPN1 - TBM differences.

Section S3: Description of other factors affecting SPN1 measurement accuracy: Thermal effects (Sect. S3.1), electrical effects (Sect. S3.2), time response (Sect S3.3) and soiling, rain, frost, snow (Sect. S3.4). Figure S6 shows illustrations of these effects.

Section S4: Detailed description of SPN1 standard firmware calculations.

Section S5: Dome lensing effect diagram.

Section S6: Diffuse first touch angle diagram. 


\section{Appendix A: Glossary}

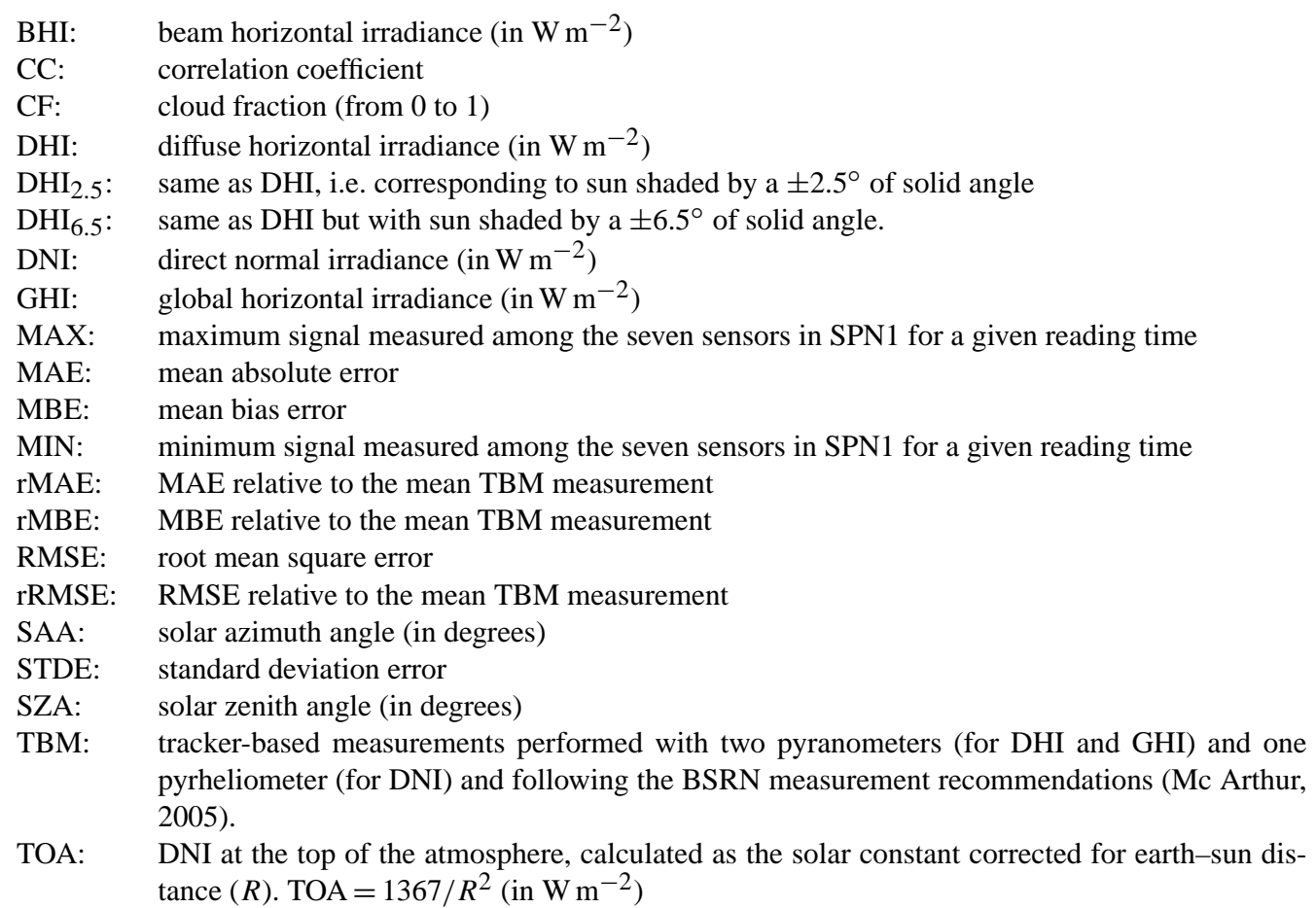




\section{The Supplement related to this article is available online at doi:10.5194/amt-7-4267-2014-supplement.}

Acknowledgements. This work was partly undertaken under the frame of the PEGASE project with the support of ADEME, EDF R\&D, ENPC, Ecole Polytechnique, and CNRS. We would like to acknowledge CEREA-EDF for providing the surface solar irradiance measurements at Roseraye and the SPN1 device at Palaiseau used in this study. We also acknowledge the contribution of the Atmospheric Radiation Measurement (ARM) Program sponsored by the U.S. Department of Energy, Office of Science, Office of Biological and Environmental Research, Climate and Environmental Sciences Division for the Addu Atol data, and NREL's Solar Radiation Research Laboratory for the Golden data. Likewise, the Payerne Baseline Surface Radiation Network station made available the data from the COST ES1002 WIRE DNI radiometer performance evaluation. Dr Long acknowledges support from the Office of Biological and Environmental Research of the U.S. Department of Energy as part of the Atmospheric Systems Research Program.

Finally, we would like to thank all those instrumentalists, often unrecognized, who provide horizontal mountings, accurate datalogging, and regular cleaning and maintenance of their sensors. Without their care many of these data would be far less useful.

Edited by: S. Schmidt

\section{References}

Delta-T Devices Ltd.: User Manual for the Sunshine Pyranometer type SPN1 UM v1.0, available at: http://www.delta-t.co.uk/ manual.html (last access: 13 July 2014), 2007.

Geuder, N., Trieb, F., Schillings, C., Meyer, R., and Quaschning, V.: Comparison of Different Methods for Measuring Solar Irradiation Data, in: 3rd International Conference on Experiences with Automatic Weather Stations, 2003.

Gueymard, C. A.: Parameterized transmittance model for direct beam and circumsolar spectral irradiance, Sol. Energy, 71, 325346, 2001.

Long, C. N., Ackerman, T. P., Gaustad, K. L., and Cole, J. N. S.: Estimation of fractional sky cover from broadband shortwave radiometer measurements, J. Geophys. Res., 111, D11204, doi:10.1029/2005JD006475, 2006.
Long, C. N., Bucholtz, A., Jonsson, H., Schmid, B., Vogelmann, A. M., and Wood, J.: A Method of Correcting for Tilt from Horizontal in Downwelling Shortwave Irradiance Measurements on Moving Platforms, Open Atmos. Sci. J., 4, 78-87, 2010.

McArthur, L. J. B.: World Climate Research Programme-Baseline Surface Radiation Network (BSRN)-Operations Manual Version 2.1, 2005.

Myers, D. R.: Comparison of direct normal irradiance derived from silicon and thermopile global hemispherical radiation detectors, paper presented at SPIE Solar Energy + Technology, International Society for Optics and Photonics, 2010.

Myers, D. R. and Wilcox, S. M.: Relative accuracy of 1-minute and daily total solar radiation data for 12 global and 4 direct beam solar radiometers, paper presented at American Solar Energy Society Annual Conference Buffalo, New York, May, 2009.

Psiloglou, B., Lykoudis, S., and Kouvas, D.: Performance Assessment of an Integrated Sensor for Simultaneous Measurements of Global and Diffuse Radiation Components at Athens Area, in: Advances in Meteorology, Climatology and Atmospheric Physics, 259-264, Springer, 2013.

Roesch, A., Wild, M., Ohmura, A., Dutton, E. G., Long, C. N., and Zhang, T.: Assessment of BSRN radiation records for the computation of monthly means, Atmos. Meas. Tech., 4, 339-354, doi:10.5194/amt-4-339-2011, 2011.

Segal-Rosenheimer, M., Russell, P. B., Livingston, J. M., Ramachandran, S., Redemann, J., and Baum, B. A.: Retrieval of cirrus properties by Sun photometry: A new perspective on an old issue, J. Geophys. Res.-Atmos., 118, 4503-4520, 2013.

Wilbert, S., Reinhardt, B., DeVore, J., Röger, M., Pitz-Paal, R., and Gueymard, C.: Measurement of solar radiance profiles with the Sun and Aureole Measurement system (SAM), 17th SolarPACES Conference, 20-23 September 2011, Granada, Spain, 2011.

Wilbert, S., Pitz-Paal, R., and Jaus, J.: Circumsolar Radiation and Beam Irradiance Measurements for Focusing Collectors, COST ES1002 WIRE Workshop on Remote Sensing Measurements for Renewable Energy, 22-23 May 2012, DTU Risoe, DK, 2012.

Wood, J. G.: Solar radiation sensor, Patent WO 99/13359, 26 pp., 1999.

WMO: CIMO Guide to meteorological instruments and methods of observation (7th Edn.) - Chapter 7: Measurement of radiation, World Meteorological Organization, WMO-No. 8 (2010 update), Geneva, Switzerland, 157-198, 2010. 